\title{
Measurements of hyperpolarizabilities for some halogenated methanes*
}

\author{
R. S. Finn ${ }^{\dagger}$ and J. F. Ward \\ The Harrison M. Randall Laboratory of Physics, University of Michigan, Ann Arbor, Michigan 48104 \\ (Received 30 July 1973) \\ de Electric-field induced optical second-harmonic generation has been measured for $\mathrm{CH}_{4}, \mathrm{CH}_{3} \mathrm{~F}$, \\ $\mathrm{CH}_{2} \mathrm{~F}_{2}, \mathrm{CHF}_{3}, \mathrm{CF}_{4}, \mathrm{CClF}_{3}$, and $\mathrm{CBrF}_{3}$. For $\mathrm{CH}_{4}$ and $\mathrm{CF}_{4}$ the third order hyperpolarizability \\ $\chi(-2 \omega ; 0, \omega, \omega)$ is obtained directly. For the other (dipolar) molecules, a bond additivity estimate for \\ $\chi(\cdots-2 \omega ; 0, \omega, \omega)$ is used to extract the second order hyperpolarizability $\chi(-2 \omega ; \omega, \omega)$
}

Results are compared with various data from the literature: calculated hyperpolarizabilities, and measurements of optical harmonic generation, optical mixing and the Kerr effect. The degree of consistency of the experimental data with a bond additivity model is discussed.

\section{INTRODUCTION}

We wish to report measurements of dc electric-field induced optical second-harmonic generation in a selection of gaseous halogenated methanes $-\mathrm{CH}_{4}, \mathrm{CH}_{3} \mathrm{~F}$, $\mathrm{CH}_{2} \mathrm{~F}_{2}, \mathrm{CHF}_{3}, \mathrm{CF}_{4}, \mathrm{CClF}_{3}$, and $\mathrm{CBrF}_{3}$. This process has been studied previously by Mayer et al. ${ }^{1,2}$ in a number of molecules, including $\mathrm{CH}_{4}$ and $\mathrm{CHF}_{3}$, among those of present interest. We havé previously reported measurements in the inert gases. ${ }^{3,4}$

Second harmonic generation by microscopically centrosymmetric systems (such as inert gas atoms) can be observed in these experiments, only when a dc electric field is present. The effect may be described in terms of the radiation from a second-harmonic dipole moment $\mathrm{p}^{2 \omega}$ given by

$$
\mathbf{p}^{2 \omega}=\frac{3}{2} \chi(-2 \omega ; 0, \omega, \omega) \mathbf{E}^{0} \mathbf{E}^{\omega} \mathbf{E}^{\omega},
$$

where $\mathbf{E}^{\omega}$ is an electric field with frequency indicated by superscript and $\chi(-2 \omega ; 0, \omega, \omega)$ is a third order hyperpolarizability. (The conventions used here in defining hyperpolarizabilities are discussed in detail in Refs. 5 and 6.) For an individual dipolar (noncentrosymmetric) molecule the second order process

$$
\mathrm{p}^{2 \omega}=\frac{1}{2} \chi(-2 \omega ; \omega, \omega) \mathrm{E}^{\omega} \mathrm{E}^{\omega}
$$

is also allowed, but is observed only by virtue of the permanent dipole moment $\mu$ interacting with the $\mathrm{dc}$ electric field to produce temperature-dependent, partial. alignment of the individual molecules. When no dc field is present, the radiation due to this process [Eq. (2)] from the randomly-oriented molecules interferes destructively and is too weak to detect in these experiments. The third order process [Eq. (1)], of course, occurs for noncentrosymmetric as well as centrosymmetric molecules.

Our goal is to extract information on the hyperpolarizabilities $\chi(-2 \omega ; 0, \omega, \omega)$ and $\chi(-2 \omega ; \omega, \omega)$ from our experimental results and to compare this with other experimental and theoretical data.

\section{EXPERIMENTAL}

The experimental apparatus is the same as that used in previous work on the inert gases ${ }^{3}$ and a schematic diagram is shown in Fig. 1. A ruby laser provides about $1 \mathrm{MW}$ of fundamental power $\left(\rho^{\omega}\right)$ at $6943 \AA$ which is brought to a focus in the gas under observation. A pair of cylindrical electrodes provides the dc electric field in the region of the focus. Second harmonic radiation at $3471 \AA$ is detected and the peak power $\left(\rho^{2 \omega}\right)$ measured, after optimizing the gas density and laser beam confocal parameter for maximum harmonic generation. It is shown in Ref. 3 for the inert gases that, under these conditions,

$$
\left|\chi(-2 \omega ; 0, \omega, \omega) / \Delta k_{0}\right|^{2} \propto \rho^{2 \omega} /\left(E^{0} \rho^{\omega}\right)^{2},
$$

where $\chi(-2 \omega ; 0, \omega, \omega)$ is a particular element of $\chi(-2 \omega$; $0, \omega, \omega) . \Delta k_{0}$ is a measure of the optical dispersion and is related to the fundamental and harmonic wave vectors $\left(k_{0}^{\omega}, k_{0}^{2 \omega}\right)$ at STP by

$$
\Delta k_{0}=2 k_{0}^{\omega}-k_{0}^{2 \omega} \text {. }
$$

For a dipolar gas, $\chi(-2 \omega ; 0, \omega, \omega)$ must be replaced by an effective hyperpolarizability $\chi^{e}(-2 \omega ; 0, \omega, \omega)$ which includes contributions from both $\chi(-2 \omega ; 0, \omega, \omega)$ and $\chi(-2 \omega ; \omega, \omega)$ as discussed in Sec. I and in more detail in Sec. III.

The experiment consists of measuring $\otimes^{\omega}, E^{0}$, and $\rho^{2 \omega}$ first for the gas under investigation, and then for helium over a total. of about 120 laser shots. $\chi^{e}(-2 \omega$; $0, \omega, \omega)$ is then extracted from Eq. (3) using $\Delta k_{0}$ from the literature $\mathrm{e}^{7}$ and using the theoretical value

$$
\chi_{y y y y}(-2 \omega ; 0, \omega, \omega) \text { helium }=3.79 \times 10^{-39} \mathrm{esu} / \text { atom }
$$

calculated by Sitz and Yaris ${ }^{\natural}$ and thought to be good to

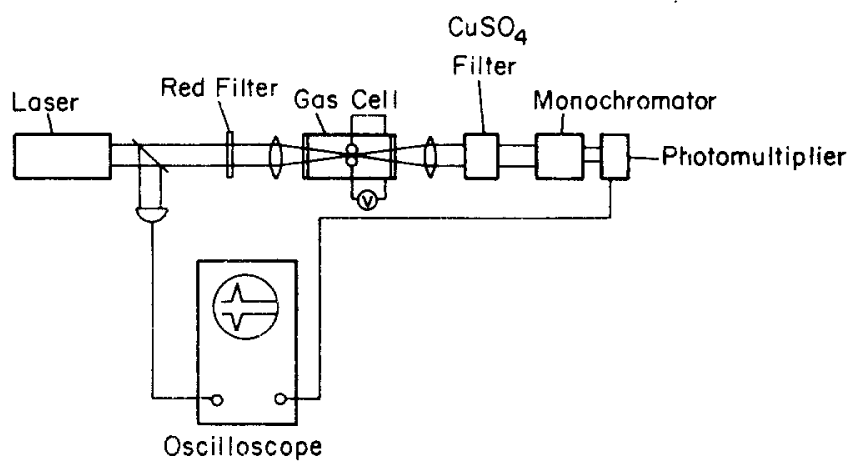

FIG. 1. Schematic diagram of the apparatus. 
TABLE I. Measured effective coefficients for do electric-field induced second-harmonic generation in halogenated methanes at $293^{\circ} \mathrm{K}$. Also given are two experimental parameters: $E_{Y}^{0}$, the maximum applied de electric field and $\rho$, the density. $\Delta k_{0}$ is the wave vector mismatch defined in Eq. 4 .

\begin{tabular}{lllll}
\hline \hline & $\begin{array}{l}\Delta k_{0}^{\mathbf{a}} \\
\mathrm{cm}^{-1}\end{array}$ & $\begin{array}{l}\rho \\
\text { amagats }\end{array}$ & $\begin{array}{l}E_{Y}^{0} \\
\text { esu }\end{array}$ & $\begin{array}{l}\chi_{Y Y Y Y}^{\mathrm{e}}(-2 \omega ; 0, \omega, \omega) \\
\left(10^{-39} \mathrm{esu} / \text { atom }\right)\end{array}$ \\
\hline $\mathrm{CH}_{4}$ & 4.05 & 1.1 & 38 & $+275 \pm 23$ \\
$\mathrm{CH}_{3} \mathrm{~F}$ & 3.59 & 1.3 & 19 & $-974 \pm 97$ \\
$\mathrm{CH}_{2} \mathrm{~F}_{2}$ & 3.05 & 1.5 & 15.2 & $-865 \pm 170$ \\
$\mathrm{CHF}_{3}$ & 2.69 & 1.7 & 30.4 & $-391 \pm 37$ \\
$\mathrm{CF}_{4}$ & 2.18 & 2.1 & 60.8 & $+101 \pm 9$ \\
$\mathrm{CClF}_{3}$ & 5.77 & 0.8 & 60.8 & $-112 \pm 7$ \\
$\mathrm{CBrF}_{3}$ & 9.43 & 0.5 & 60.8 & $-489 \pm 24$ \\
\hline \hline
\end{tabular}

${ }^{\mathrm{a}}$ Reference 7 .

1\%. Data for the halogenated methanes are shown in Table I.

The signs of the measured hyperpolarizabilities are important in further analysis of the data (Sec. IV). We have measured relative signs by studying dc electricfield induced second-harmonic generation in various binary mixtures of gases. Knowing $\Delta k_{0}$ and the magnitude $\left|\chi^{e}(-2 \omega ; 0, \omega, \omega)\right|$ for each of two gases (labelled 1 and 2 below), a partial density for each $\left(\rho_{1}, \rho_{2}\right)$ was calculated such that the mixture would give optimum harmonic generation if $\chi_{1}^{e}(-2 \omega ; 0, \omega, \omega)$ and $\chi_{2}^{e}(-2 \omega ; 0, \omega, \omega)$ were of the same sign but zero harmonic generation if they have opposite signs. The experiment was then performed with the density of gas 2 fixed at $\rho_{2}$ while that of gas 1 was varied in the range 0 to $3 \rho_{1}$. The results for mixtures of $\mathrm{CHF}_{3}$ and $\mathrm{CH}_{4}$ are shown in Fig. 2 together with the two alternative theoretical curves. Occasional failure of the data to follow either curve was traced to inadequate mixing of the gases. To determine the absolute signs for hyperpolarizabilities shown in Table I, we further assume that for argon the sign of $\chi(-2 \omega$; $0, \omega, \omega)$ is the same as that of $\chi(-\omega ; 0,0, \omega)$ the Kerr coefficient. ${ }^{9}$ This will be justified by arguments at the end of Sec. III which indicate that the sign and magnitude of these two hyperpolarizabilities should be approximately equal.

\section{HYPERPOLARIZABILITIES IN THE MOLECULAR AND LABORATORY COORDINATE FRAMES}

We wish to relate the measured, effective hyperpolarizability $\chi^{e}(-2 \omega ; 0, \omega, \omega)$ to the third and second order molecular hyperpolarizabilities $\chi(-2 \omega ; 0, \omega, \omega)$ and $\chi(-2 \omega ; \omega, \omega)$. The analogous problem in the Kerr effect has been treated by Buckingham ${ }^{10}$, results for dc electric-field induced second-harmonic generation have been given by Mayer et al. ${ }^{2}$ and by Kielich. ${ }^{11}$ We outline the derivation here to introduce our notation and review the approximations involved.

The total molecular dipole moment at the second harmonic frequency is obtained by combining Eqs. (1) and (2):

$$
p_{i}^{2 \omega}=\frac{1}{2} \chi_{i k l}(-2 \omega ; \omega, \omega) E_{k}^{\omega} E_{l}^{\omega}
$$

$$
+\frac{3}{2} \chi_{i j k l}(-2 \omega ; 0, \omega, \omega) E_{j}^{0} E_{k}^{\omega} E_{l}^{\omega} \ldots,
$$

Where coordinate subscripts $i, j, k, l$ refer to $x, y, z$ in the coordinate frame of the molecule. The effective hyperpolarizability can be defined in terms of $\overline{p^{2 \omega}}$, the average harmonic dipole moment per molecule in the laboratory coordinate frame (indicated by capitalized subscripts $F, G, H, M$ referring to $X, Y, Z$ ):

$$
\overline{p_{F}^{2 \omega}}=\frac{3}{2} \chi_{F G H}^{e}(-2 \omega ; 0, \omega, \omega) E_{G}^{0} E_{H}^{\omega} E_{M}^{\omega} \ldots
$$

In the present experiments, there is negligible difference between applied and local fields. Also, it can be shown that intermolecular interactions may be neglected. ${ }^{4}$ Thus the average of $p_{i}^{2 \omega}$ to yield $\overline{p_{F}^{2 \omega}}$ is taken over orientations of molecules with a Boltzmann weighting factor according to the interaction energy of the molecular permanent dipole moment $\mu$ with the applied dc field $E_{G}^{0}$. This classical approach is adequate for the se molecules at ambient temperatures. The weakness of the alignment produced by the dc electric field allows expansion of the Boltzmann weighting factors to yield the result in terms of an isotropic orientational average (indicated by angular brackets \langle\rangle ):

$$
\begin{aligned}
\overline{p_{F}^{2 . \omega}}= & \frac{3}{2}\left\langle\Phi_{F i} \Phi_{G j} \Phi_{H k} \Phi_{M l}\right\rangle \\
& \times\left[\chi_{i j k l}(-2 \omega ; 0, \omega, \omega)\right. \\
& \left.+\left(\mu_{j} / 3 k T\right) \chi_{i k l}(-2 \omega ; \omega, \omega)\right] E_{G}^{0} E_{H}^{\omega} E_{M}^{\omega}
\end{aligned}
$$

where $\Phi_{F i}$ is the direction cosine between the laboratory $F$ direction and the molecular $i$ direction. Averages of products of $\Phi$ 's have been conveniently tabulated by Cyvin et al. ${ }^{12}$ Comparing Eq. (8) with (7) we can separate the effective hyperpolarizability into thi rd order and second order contributions:

$$
\begin{aligned}
& \chi_{F G H M}^{e}(-2 \omega ; 0, \omega, \omega) \\
& \quad \equiv \bar{\chi}_{F G H}(-2 \omega ; 0, \omega, \omega)+(\mu / 9 k T) \bar{\chi}_{F G H M}(-2 \omega ; \omega, \omega)
\end{aligned}
$$

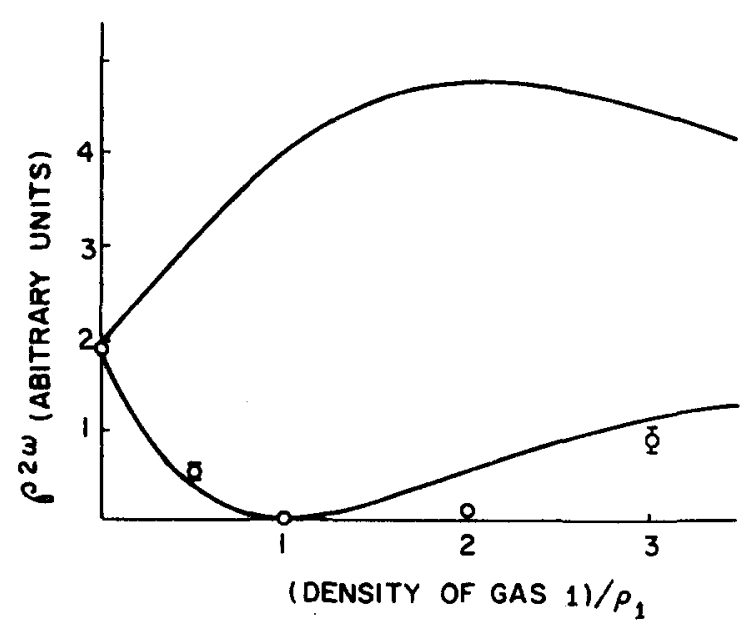

FIG. 2. Harmonic generation in a mixture of gas $1\left(\mathrm{CHF}_{3}\right)$ and gas $2\left(\mathrm{CH}_{4}\right)$. The density of gas 1 is varied in the range 0 to $3 \rho_{1}$ while the density of gas 2 is kept at $\rho_{2}$. The values $\rho_{1}$ and $\rho_{2}$ are discussed in the text. The two theoretical curves are computed for two gases with $\chi^{e}(-2 \omega ; 0, \omega, \omega)$ of the same sign (upper curve) and opposite sign (lower curve). The data in this instance clearly follow the lower curve. 
TABLE II. Third order hyperpolarizabilities in units of $\left(10^{-39}\right.$ esu/atom). Results from the present experiment are shown in the first column. Estimates for dipolar molecules derived from the bond additivity model are given in the second column based on data for the tetrahedral molecules shown in parentheses. Other experimental data are also shown.

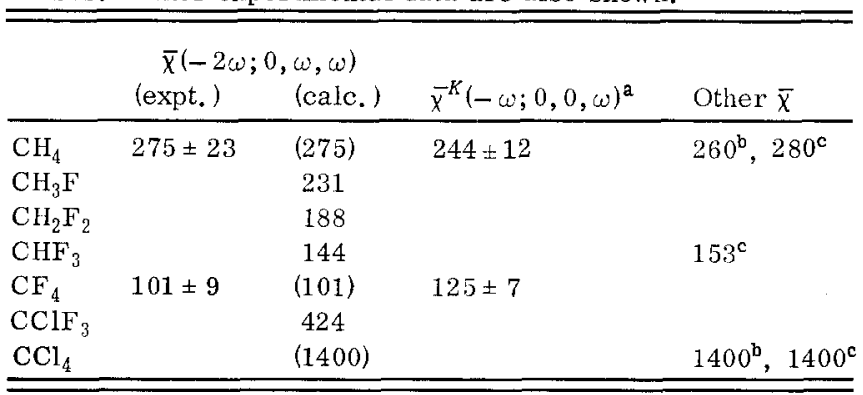

${ }^{2}$ Reference 13 .

$\mathrm{b} \bar{\chi}(-2 \omega ; 0, \omega, \omega)$ from Ref. 2 .

${ }^{\complement} \bar{\chi}\left(-2 \omega+\omega_{s} ;-\omega_{s}, \omega, \omega\right)$ from Ref. 2 .

and then relate each of these to corresponding molecular hyperpolarizabilities:

$\vec{\chi}_{F G H M}(-2 \omega ; 0, \omega, \omega)=\left\langle\Phi_{F i} \Phi_{G j} \Phi_{H k} \Phi_{M l}\right\rangle \chi_{i j k l}(-2 \omega ; 0, \omega, \omega)$

and

$$
\bar{\chi}_{F G H M}(-2 \omega ; \omega, \omega)=\left\langle\Phi_{F i} \Phi_{G z} \Phi_{H k} \Phi_{M l}\right\rangle \chi_{i k l}(-2 \omega ; \omega, \omega) .
$$

Note the subscript $z$ on the second direction cosine in Eq. (11) which appears because the molecular $z$ axis has been placed along the permanent dipole. Also in Eqs. (9) and (11) we are using the notation $\bar{\chi}_{F G H M}(-2 \omega ; \omega, \omega)$ to indicate the linear combination of molecular hyperpolarizability elements with frequency labels $(-2 \omega ; \omega, \omega)$ which contributes in an experiment when the induced dipole moment and the three applied fields are in the laboratory frame $F, G, H$ and $M$ directions respectively.

For spherically symmetric molecules, $\mu$ and $\chi(-2 \omega$; $\omega, \omega)$ are zero and there is no distinction among $\chi_{F G H M}^{e}$ $\times(-2 \omega ; 0, \omega, \omega), \bar{\chi}_{F G H M}(-2 \omega ; 0, \omega, \omega)$, and $\chi_{i j k l}(-2 \omega ; 0, \omega, \omega)$ since the laboratory coordinate frame can serve as an equivalent molecular frame for all molecules. Tetrahedral molecules are not centrosymmetric but have no dipole moments and the second order hyperpolarizability does not contribute to $\chi^{e}(-2 \omega ; 0, \omega, \omega)$.

In Sec. IV, our results will be compared with data from the literature for two other processes, and it is convenient to display for them equations analogous to Eqs. (9), (10), and (11). Firstly, a mixing process with hyperpolarizability $\chi\left(-2 \omega+\omega_{s} ;-\omega_{s}, \omega, \omega\right)$, which has been investigated by Mayer et al., ${ }^{2}$ differs from dc electricfield induced second-harmonic generation in that the dc field has been replaced by an infrared field with frequency $\omega_{s}$. The infrared field varies too rapidly to align molecular dipoles so that the second order coefficient does not contribute to the process:

$$
\begin{gathered}
\chi_{F G H M}^{e}\left(-2 \omega+\omega_{s} ;-\omega_{s}, \omega, \omega\right)=\bar{\chi}_{F G H M}\left(-2 \omega+\omega_{s} ;-\omega_{s}, \omega, \omega\right) \\
=\left\langle\Phi_{F i} \Phi_{G j} \Phi_{H k} \Phi_{M I}\right\rangle \chi_{i j k l}\left(-2 \omega+\omega_{s} ;-\omega_{s}, \omega, \omega\right) .
\end{gathered}
$$

Secondly, the Kerr effect has been studied by Bucking- ham and $\operatorname{Orr}^{13}$ and corresponding results for the first virial coefficient $A_{k}$ are:

$$
\begin{aligned}
A_{k}= & \frac{8}{27} \pi N_{0}\left[\left\langle\Phi_{F i} \Phi_{F k} \Phi_{F j} \Phi_{F l}\right\rangle-\left\langle\Phi_{G i} \Phi_{G k} \Phi_{F j} \Phi_{F l}\right\rangle\right] \\
& \times\left[\frac{3}{2}\left\{\chi_{i j}{ }_{l k}(-\omega ; 0,0, \omega)+\left(2 \mu_{l} / 3 k T\right) \chi_{i j k}(-\omega ; 0, \omega)\right\}\right. \\
& \left.+\frac{1}{4}\left\{(k T)^{-1} \chi_{i k}(-\omega ; \omega) \chi_{j l}(0 ; 0)+(k T)^{-2} \chi_{i k}(-\omega ; \omega) \mu_{j} \mu_{l}\right\}\right] \\
= & \frac{8}{27} \pi N_{0}\left[\left\{\bar{\chi}^{K}(-\omega ; 0,0, \omega)+\frac{2}{9}(k T)^{-1} \mu \bar{\chi}^{K}(-\omega ; 0, \omega)\right\}\right. \\
& \left.+\left\{\text { terms in } \chi_{i k}(-\omega ; \omega) \text { etc }\right\}\right],
\end{aligned}
$$

where $N_{0}$ is Avogadro's number,

$$
\begin{aligned}
\bar{\chi}^{K}(-\omega ; 0,0, \omega)= & \frac{3}{2}\left[\left\langle\Phi_{F i} \Phi_{F k} \Phi_{F j} \Phi_{F l}\right\rangle-\left\langle\Phi_{G i} \Phi_{G k} \Phi_{F j} \Phi_{F l}\right\rangle\right] \\
& \times \chi_{i j l k}(-\omega ; 0,0, \omega)
\end{aligned}
$$

and

$$
\begin{aligned}
\bar{\chi}^{K}(-\omega ; 0, \omega)= & {\left[\left\langle\Phi_{F i} \Phi_{F k} \Phi_{F j} \Phi_{F z}\right\rangle-\left\langle\Phi_{G i} \Phi_{G k} \Phi_{F j} \Phi_{F z}\right\rangle\right] } \\
& \times \chi_{i j k}(-\omega ; 0, \omega) .
\end{aligned}
$$

The isotropic averaging in Eqs. (10)-(15) imposes spherical symmetry on the laboratory frame coefficients so that $\chi^{e}(-2 \omega ; 0, \omega, \omega)$, for example, has only two nonzero independent components which may be chosen to be those with coordinate subscripts $Y Y Y Y$ and $Y Y X X$. Each laboratory frame coefficient is a different linear combination of molecular coefficients as prescribed by Eqs. (10), (11), (12), (14), and (15). A molecular coefficient depends on the frequencies of the fields involved and is subject to restrictions imposed by molecular symmetry and (far from resonance) by permutation symmetry. ${ }^{14}$

A sweeping simplification occurs in the zero frequency limit $\left(\omega, \omega_{s} \rightarrow 0\right)$. In this limit, the third order hyperpolarizabilities obey the relations

$$
\begin{aligned}
\bar{\chi}_{Y Y Y Y}(-2 \omega ; 0, \omega, \omega) & =3 \bar{\chi}_{Y Y X X}(-2 \omega ; 0, \omega, \omega) \\
& =\bar{\chi}_{Y Y Y Y}\left(-2 \omega+\omega_{s} ;-\omega_{s}, \omega, \omega\right) \\
& =3 \chi_{Y Y X X}\left(-2 \omega+\omega_{s} ;-\omega_{s}, \omega, \omega\right) \\
& =\bar{\chi}^{K}(-\omega ; 0,0, \omega)=\gamma / 6,
\end{aligned}
$$

and similarly for the second order hyperpolarizabilities: $\bar{\chi}_{Y Y Y Y}(-2 \omega ; \omega, \omega)=3 \bar{\chi}_{Y Y X X}(-2 \omega ; \omega, \omega)=\bar{\chi}^{K}(-\omega ; 0, \omega)=\beta / 2$,

where $\gamma$ and $\beta$ are notations often used in the literature. ${ }^{15}$ We expect the zero frequency limit to be a useful approximation here since all applied field frequencies are small compared with the frequencies of excitations which dominate the linear optical properties of these molecules. Our experiments have given direct evidence of consistency with one feature of these predictions

$\left|\chi_{Y Y Y Y}^{e}(-2 \omega ; 0, \omega, \omega) / 3 \chi_{Y Y X X}^{e}(-2 \omega ; 0, \omega, \omega)-1\right| \leq 0.1$

Hereafter we shall not be directly concerned with $Y Y X X$ components and the set of coordinate subscripts $Y Y Y Y$ will be suppressed for brevity.

\section{RESULTS AND DISCUSSION}

\section{A. Third order hyperpolarizabilities}

In the case of tetrahedral molecules $\left(\mathrm{CH}_{4}, \mathrm{CF}_{4}\right.$, $\mathrm{CCl}_{4}$ ) the dipole moment is zero and experiments yield 
TABLE III. Second order hyperpolarizabilities in units of $\left(10^{-31} \mathrm{esu} /\right.$ atom $)$.

\begin{tabular}{lllll}
\hline \hline & $\begin{array}{l}\mu^{\mathrm{a}} \\
\left(10^{-18} \text { esu) }\right.\end{array}$ & $\begin{array}{l}\bar{\chi}(-2 \omega ; \omega, \omega) \\
\text { (expt) }\end{array}$ & $\begin{array}{l}\bar{\chi}^{K}(-\omega ; 0, \omega)^{\mathrm{c}} \\
\text { (expt) }\end{array}$ & $\begin{array}{l}\vec{\chi}(0 ; 0,0) \\
\text { (calc) }\end{array}$ \\
\hline $\mathrm{CH}_{4}$ & 0 & 0 & 0 & \\
$\mathrm{CH}_{3} \mathrm{~F}$ & 1.85 & $-2.37 \pm 0.20$ & $-2.5 \pm 1.32$ & $-0.259^{\mathrm{d}}$ \\
& & & & $-0.800^{\mathrm{\theta}}$ \\
$\mathrm{CH}_{2} \mathrm{~F}_{2}$ & 1.97 & $-1.95 \pm 0.32$ & $-0.55 \pm 0.13$ & $-0.342^{\mathrm{d}}$ \\
$\mathrm{CHF}_{3}$ & 1.65 & $-1.18 \pm 0.10$ & $+3.64 \pm 1.35$ & $+0.302^{\mathrm{d}}$ \\
$\mathrm{CF}_{4}$ & 0 & $-0.74^{\mathrm{b}}$ & & \\
$\mathrm{CClF}_{3}$ & 0.50 & 0 & $-3.90 \pm 0.47$ & \\
$\mathrm{CBrF}_{3}$ & 0.65 & & & \\
\hline \hline
\end{tabular}

${ }^{a}$ R. D. Nelson, Jr., D. R. Lide, Jr., and A. A. Maryott, Selected Values of Electric Dipole Moments for Molecules in the Gas Phase [Natl. Stand. Ref. Data Ser. 10 (1967)].

${ }^{b}$ Reference 2.

${ }^{c}$ Reference 13.

${ }^{\mathrm{d}}$ Reference 17 .

${ }^{\circ}$ Reference 16.

the thi rd order hyperpolarizability directly. Table II shows results from de electric-field induced secondharmonic generation (Mayer el al. ${ }^{2}$ in addition to the present work), the Kerr effect (Buckingham and Or ${ }^{13}$ ), and an optical mixing process (Mayer et al. ${ }^{2}$ ). The coefficients for different processes in a particular molecule can be seen to be equal to within about $\pm 20 \%$ which is the level of experimental uncertainty. Thus the data is consistent with the prediction of the zero frequency limit, Eq. (16).

Table II also shows estimates of $\bar{\chi}(-2 \omega ; 0, \omega, \omega)$ for the dipolar gases calculated using the bond additivity model. ${ }^{15}$ In this model, a hyperpolarizability is ascribed to each bond from which the molecular hyperpolarizability can be determined by geometric combination. Our experimental results for $\mathrm{CH}_{4}$ and $\mathrm{CF}_{4}$ and results for $\mathrm{CCl}_{4}$ from Ref. (2) have been used to give the hyperpolarizabilities for $\mathrm{CH}, \mathrm{CF}$, and $\mathrm{CCl}$ bonds and, in turn, predict the third order hyperpolarizabilities for the dipolar molecules. In the case of $\mathrm{CHF}_{3}$, the reliability of the bond additivity estimate can be tested by comparison with the data of Mayer et al. ${ }^{2}$ The third order hyperpolarizabilities have been shown to be consistent with the zero frequency limit. Thus the experimental value of $\bar{\chi}\left(-2 \omega+\omega_{s} ; \omega_{s}, \omega, \omega\right)$ should provide a good estimate for $\bar{\chi}(-2 \omega ; 0, \omega, \omega)$ and is within $7 \%$ of the bond additivity estimate of the same quantity. We therefore believe that the bond additivity model provides estimates of third order hyperpolarizabilities which are good to within $\pm 15 \%$.

\section{B. Second order hyperpolarizabilities}

For dipolar molecules, the experimental results depend on both third and second order hyperpolarizabilities [see Eq. (9)]. We therefore follow Buckingham and Orr ${ }^{13}$ in using the bond additivity estimate for $\bar{\chi}(-2 \omega$; $0, \omega, \omega)$ from Table II and a knowledge of $\mu$ to extract $\bar{\chi}(-2 \omega ; \omega, \omega)$ from the experiment. Values for $\bar{\chi}(-2 \omega$; $\omega, \omega)$ derived in this way are shown in Table III together with uncertainties which include the experimental uncertainties in $\chi^{e}(-2 \omega ; 0, \omega, \omega)$ and an estimated uncertainty of $\pm 15 \%$ for the bond additivity value of $\bar{\chi}(-2 \omega$; $0, \omega, \omega)$. The latter contribution is unimportant except in the case of $\mathrm{CClF}_{3}$.

Buckingham and Orr ${ }^{13}$ extract $\bar{\chi}^{K}(-\omega ; 0, \omega)$ from Kerr effect data [see Eq. (13)] making use of the temperature dependence of the Kerr effect, known values of dipole moments and linear polarizabilities and using the bond additivity approximation to estimate $\bar{\chi}^{K}(-\omega ; 0,0, \omega)$. Their results are also shown in Table III. Mayer et al. ${ }^{2}$ have derived $\bar{\chi}(-2 \omega ; \omega, \omega)$ for $\mathrm{CHF}_{3}$ (and for other molecules not of direct interest here) from the difference between $\chi^{e}(-2 \omega ; 0, \omega, \omega)$ and $\bar{\chi}\left(-2 \omega+\omega_{s} ;-\omega_{s}, \omega, \omega\right)$, assuming the latter is equal to $\bar{\chi}(-2 \omega ; 0, \omega, \omega)$ [see Eqs. (9) and (12)]. This result is also shown in Table III. Values for dc hyperpolarizabilities $\chi(0 ; 0,0)$ calculated by Arrighini et al. ${ }^{16}$ and by Hush and William ${ }^{17}$ are included in Table III.

For $\mathrm{CHF}_{3}$ the present value and that of Mayer are in satisfactory agreement. However, as noted by Mayer, the result from the Kerr coefficient measurement is three times as large and of opposite sign. We find such a large deviation from the low frequency limit prediction of equality [see Eq. (17)] to be surprising. The other experimental results show less striking disagreement. The theoretical values have the same signs as those from the Kerr measurements, but their magnitudes are all significantly smaller than any of the measured hyperpolarizabilities for the same molecule.

Comparison of the data from Table III with bond additivity estimates for the second order hyperpolarizabilities is shown in Table IV. Again for $\mathrm{CHF}_{3}$, the value from the Kerr measurement shows surprisingly gross deviation from the predicted value. Buckingham and Orr ${ }^{13}$ have commented on the basis of their Kerr data: "The lack of agreement does not augur well for the success of a bond additivity approximation in predicting second order hyperpolarizabilities for molecules." We would be much more optimistic with only the second harmonic data as a guide, characterizing the bond additivity approach as useful but imprecise. A bond additivity approximation for second order hyperpolarizabilities is particularly attractive since it is an integral part of Levine's method ${ }^{18}$ of calculation which has been so successful for a wide range of crystals.

The state of understanding of second order hyperpolarizabilities for the halogenated methanes as summarized in Table III is clearly not yet satisfactory.

We plan to refine our dc electric-field induced second-

TABLE IV. Second order hyperpolarizabilities from Table III, normalized to $\mathrm{CH}_{3} \mathrm{~F}$ and compared with the predictions of the bond additivity model.

\begin{tabular}{llcl}
\hline \hline & $\bar{\chi}(-2 \omega ; \omega, \omega)$ & $\bar{\chi}^{K}(-\omega ; 0, \omega)$ & $\begin{array}{l}\text { Bond Additivity } \\
\text { Model }\end{array}$ \\
\hline $\mathrm{CH}_{3} \mathrm{~F}$ & 1.00 & 1.00 & 1.00 \\
$\mathrm{CH}_{2} \mathrm{~F}_{2}$ & $0.82 \pm 0.06$ & $0.22 \pm 0.13$ & 0.87 \\
$\mathrm{CHF}_{3}$ & $0.50 \pm 0.06$ & $-1.46 \pm 0.94$ & 1.00 \\
\hline \hline
\end{tabular}


harmonic data by measuring coefficients as a function of temperature. The experiment will then yield [see Eq. (9)] both $\bar{\chi}(-2 \omega ; 0, \omega, \omega)$ and $\bar{\chi}(-2 \omega ; \omega, \omega)$ directly from the data for each dipolar gas without the need to use bond additivity estimates for the third order hyperpolarizabilities.

\section{ACKNOWLEDGMENT}

It is a pleasure to acknowledge helpful discussions with Irving J. Bigio.

*This research was supported in part by the Air Force Office of Scientific Researeh under Grant 72-2302.

$\dagger$ Present address: General Electric Company, G. E. TEM PO, P. O. Drawer QQ, Santa Barbara, California 93102.

'G. Mayer, C. R. Acad. Sci. B 267, 54 (1968).

${ }^{2} \mathrm{G}$. Hauchecorne, F. Kerherve, and G. Mayer, J. Phys. (Paris) 32, 47 (1971). In quoting data from this reference we have used the alternative calibration discussed by the authors. This involves normalizing with respect to the measured Kerr coefficient for argon (see Ref. 13) and seems to be more consistent with other norrnalizations involved in data discussed here.

${ }^{3}$ R. S. Finn and J. F. Ward, Phys, Rev, Lett, 26, 285 (1971).

${ }^{4}$ R. S. Finn, "Optical de-induced Second Harmonic Generation in Gases," Thesis, University of Michigan, 1971 (unpublished).

${ }^{5}$ J. F. Ward and G. H. C. New, Phys, Rev. 185, 57 (1969).

${ }^{6}$ B. J. Orr and J. F. Ward, Mol. Phys. 20, 513 (1971),

${ }^{7}$ R. S. Finn and J. F. Ward, J. Appl. Opt. 11, 2103 (1972).

${ }^{8}$ P. Sitz and R. Yaris, J. Chem. Phys, 49, 3546 (1968).

${ }^{9}$ A. D. Buckingham and D. A. Dunmur, Trans. Faraday Soc. 64,1776 (1968).

${ }^{10}$ A. D. Buckingham and J. A. Pople, Proc. Phys. Soc. Lond. A 68, 905 (1955); A. D. Buckingham, Proc. Phys. Soc. Lond. A 68,910 (1955).

"S. Kielich, IEEE J. Quantum Electron. QE5, 562 (1969).

${ }^{12}$ S. J. Cyvin, J. E. Rauch, and J. C. Decius, J. Chem. Phys. 43, 4083 (1965).

${ }^{13}$ A. D. Buckingham and B. J. Orr, Trans. Faraday Soc. 65, 673 (1969).

${ }^{14}$ P. S. Pershan, Phys. Rev. 130, 919 (1963).

${ }^{15} \mathrm{~A}$, D. Buckingham and B. J. Orr, Q. Rev. Chem. Soc. 21, 195 (1967).

${ }^{16}$ G. P. Arrighini, M. Maestro, and R. Moccia, Symp. Faraday Soc. 2, 48 (1968).

${ }^{17}$ N. S. Hush and M. L. Williams, Theoret. Chim. Acta. 25, 346 (1972).

${ }^{18}$ B. F. Levine, Phys. Rev. B 7, 2600 (1973). 